\title{
Genome-wide identification, evolution, and expression analysis of the NPR1-like gene family in pears
}

\author{
Yarui Wei ${ }^{1}$, Shuliang Zhao ${ }^{2}$, Na Liu ${ }^{1}$, Yuxing Zhang ${ }^{\text {Corresp. } 1}$ \\ ${ }^{1}$ Hebei Agricultural University, College of Horticulture, Baoding, Hebei, China \\ 2 Hebei University of Engineering, School of Landscape and Ecological Engineering, Handan, Hebei, China \\ Corresponding Author: Yuxing Zhang \\ Email address: zhyx@hebau.edu.cn
}

The NONEXPRESSOR OF PATHOGENESIS-RELATED GENES 1 (NPR1) plays a master regulatory role in the salicylic acid (SA) signal transduction pathway and plant systemic acquired resistance (SAR). Members of the NPR1-like gene family have been reported to the associated with biotic/abiotic stress in many plants, however the genome-wide characterization of NPR1-like genes has not been carried out in Chinese pear (Pyrus bretschneideri Reld). In this study, a systematic analysis was conducted on the characteristics of the NPR1-like genes in P. bretschneideri Reld at the whole-genome level. A total nine NPR1-like genes were detected of which eight genes were located on six chromosomes and one gene was mapped to scaffold. Based on the phylogenetic analysis, the nine PbrNPR1-like genes were divided into three clades (Clades I-III) has similar gene structure, domain and conserved motifs. We sorted the cis-acting elements into the three clades, including plant growth and development, stress responses, and hormone responses in promoter regions of PbrNPR1-like genes. The result of qPCR analysis showed that expression diversity of PbrNPR1-like genes in various tissues. All the genes were upregulated after SA treatment in leaves except for Pbrgene8896. PbrNPR1-like genes showed circadian rhythm and significantly different expression levels after inoculation with Alternaria alternata. These findings provide a solid insight for understanding the functions and evolution of PbrNPR1-like genes in Chinese pear 


\title{
Genome-wide Identification, Evolution, and Expression Analysis of the NPR1-like Gene Family in Pears
}

\author{
Yarui Wei ${ }^{1}$, Shuliang Zhao ${ }^{2}$, Na Liu ${ }^{1}$, Yuxing Zhang ${ }^{1 *}$. \\ ${ }^{1}$ College of Horticulture, Hebei Agricultural University, Baoding 071001, Hebei, China; \\ ${ }^{2}$ School of Landscape and Ecological Engineering, Hebei University of Engineering, Handan \\ 056038, Hebei, China. \\ Corresponding Author: \\ Yuxing Zhang* \\ Leka Street, Baoding, Heibei, China \\ Email address: zhyx@hebau.edu.cn
}

\section{Abstract}

The NONEXPRESSOR OF PATHOGENESIS-RELATED GENES 1 (NPR1) plays a master regulatory role in the salicylic acid (SA) signal transduction pathway and plant systemic acquired resistance (SAR). Members of the NPRI-like gene family have been reported to the associated with biotic/abiotic stress in many plants, however the genome-wide characterization of NPRIlike genes has not been carried out in Chinese pear (Pyrus bretschneideri Reld). In this study, a systematic analysis was conducted on the characteristics of the NPRl-like genes in $P$. bretschneideri Reld at the whole-genome level. A total nine NPRI-like genes were detected of which eight genes were located on six chromosomes and one gene was mapped to scaffold. Based on the phylogenetic analysis, the nine PbrNPR1-like genes were divided into three clades (Clades I-III) has similar gene structure, domain and conserved motifs. We sorted the cis-acting elements into the three clades, including plant growth and development, stress responses, and hormone responses in promoter regions of $P b r N P R 1$-like genes. The result of qPCR analysis showed that expression diversity of $P b r N P R 1$-like genes in various tissues. All the genes were up-regulated after SA treatment in leaves except for Pbrgene8896. PbrNPR1-like genes showed circadian rhythm and significantly different expression levels after inoculation with Alternaria alternata. These findings provide a solid insight for understanding the functions and evolution of PbrNPRI-like genes in Chinese pear.

Keywords: NPR1; SAR; phylogenetic; expression; salicylic acid.

\section{Introduction}

Pears are threatened with various diseases which were caused by fungus, bacterial, viruses, nematodes and insect bites in environment (Robert-Seilaniantz et al. 2011). These diseases limit pear quality and generate severe economic loss. Changes in phytohormones concentration or sensitivity can be triggered under biotic and abiotic stress conditions (Pieterse et al. 2009). Salicylic acid (SA) signaling triggers the resistance against biotrophic and hemibiotrophic 
40 pathogens (Nie et al. 2017), whereas a combination of Jasmonates ( JA) and Ethylene (ET)

41 signaling activates the resistance against necrotrophic pathogens (Attaran \& He 2012). These

42 two pathways are mostly antagonistic: elevated biotroph resistance is often correlated with

43 increased necrotroph susceptibility, while elevated necrotroph resistance is correlated with

44 enhanced susceptibility to biotrophs (Robert-Seilaniantz et al. 2011). SA has been found to be an

45 essential in systemic acquired resistance (SAR), a broad-spectrum and long lasting plant immune

46 response to pathogens (An \& Mou 2011). In SAR mediated immune response,

47 NONEXPRESSOR OF PATHOGENESIS-RELATED GENES 1 (NPR1) is responsible for

48 positive regulation. $P R 1$ gene is a molecular marker for SAR: in unchallenged cells, its

49 transcription is repressed by TGA2 transcription factor (Cao et al. 1998). In transgenic

50

51

52

53

54

55

56

57

58

59

60

61

62

63

64

65

66

67

68

69

70

71

72

73

74

75

76

77

78

79

Arabidopsis thaliana (A.thaliana) overexpressed the $n a h G$ gene encodes salicylate hydroxylase to prevent pathogen-induces accumulation of SA content and the activation of SAR. The plants showed an increase in endogenous SA, and subsequently hypersensitive response (HR) at the site of infection (Lawton et al. 1995).

As SA receptor, NPR1 is a transcription co-activator (Wu et al. 2012). The NPR1 gene encodes a protein with two protein-protein interaction domains, ankyrin repeat domain (ANK) and BTB/POZ domain (Broad Complex, Tramtrack, and Bric a Brac/Pox virus and Zinc finger). It also has a transcriptional activation domain and a nuclear localization sequence in the $\mathrm{C}$ terminal (Dong 2004). The BTB/POZ domain is beneficial to dimerize of NPR1, and the ankyrin-repeat domain interacts with the TGA of bZIP transcription factors (Boyle et al. 2009; Rochon et al. 2006). NPR1 protein localizes both in the nucleus and cytoplasm. Before the pathgen infection, the content of SA is low in the plant, and NPR1 localizes in the cytoplasm as inactive oligomer through disulfide bonds. When a pathogen infects plant, the endogenous SA increases and the redox state changes in the cytoplasm, and NPR1 changes to active monomer, transferring from the cytoplasm to the nucleus. As transcriptional co-activator, NPR1 interacts with TGA to regulate downstream gene expression. Then PR gene expression increases and induces SAR (Kinkema et al. 2000; Mou et al. 2003).

NPR1 and its paralogues NPR3 and NPR4, were SA receptors that undertake as dominant regulators in SA-mediated local resistance and SAR (Robert-Seilaniantz et al. 2011). In transgenic apple (Fuji), overexpressing MhNPRl gene induced SAR and exhibited enhanced resistance to fungal diseases (Chen et al. 2012). Using the CRISPER/Cas9 system, Slnpr 1 mutants displayed reduced tomato drought tolerance (Li et al. 2019). Ectopic expression of $M u N P R 1$ showed enhanced scavenging ability and suppressed collapse accumulation, whereas the MuNPR4 transgenic Arabidopsis were hypersensitive to Pseudomonas syringae pv. tomato. DC3000 (Pst.DC3000) infection (Xu et al. 2019). Recently, a new mechanism was revealed that NPRI facilitated its own expression by recruiting WRKY18 and CDK8 to the promoter of NPR1, leading the increased expression of the $P R$ gene (Chen et al. 2019). NPR1, as SA receptor, involved in the chitosan-induced stomata closure and thus played a vital role to adjust to the adverse environments in plants (Prodhan et al. 2020). The proteins NPR3 and NPR4 bind SA and their function is transcriptional co-repressor and they are partly redundant in their function (Fu et 
80

81

82

83

84

85

86

87

88

89

90

91

92

93

94

95

96

97

98

99

100

101

102

103

104

105

106

107

108

109

110

111

112

113

114

115

116

117

118

119

al. 2012). FvNPRL-1 was found closer to NPR3/NPR4 in A.thaliana, and ectopic expression of FvNPRL-1 in wild type of A.thaliana suppressed the resistance to Pst.DC3000 (Wang et al. 2018).

Focusing on the function of redox rhythm and NPR1 in the plant immunity and circadian clock (Zhang et al. 2019) proved the complex relationship between plant immune response and circadian clock. Plant circadian clocks play an important role in regulating the growth-defense balance. The nocturnal stomatal closure and active defense in the morning are good examples of how the circadian clock stop the unsustainable energy shift to immunity. Through the interaction with the circadian redox rhythm of metabolism, the circadian clock shuts off immune induction to prevent conflict with growth at night (Dodd et al. 2005; Somers et al. 1998). The circadian clock combines environmental cues with temporal information to regulate the plant physiology and development.

In A.thaliana, $N P R 1$-like gene family has six members $N P R 1, N P R 1$-like 2 (NPR2), NPR3, NPR4, BLADE-ON- PETIOLE2 (BOP2; NPR5), and BOP1 (NPRO). NPR1, NPR2, NPR3, NPR4 participate in the SA signal transduction pathway (Hepworth et al. 2005; McKim et al. 2008). Using bioinformatics methodes, there were 17, 12, 5, and 6 NPR1-like genes in Triticum aestivum, Triticum dicoccoides, Triticum urartu, Aegilops tauschii, respectively (Liu et al. 2019). Five NPRI-like genes were discovered in Persea americana (Mill) which harbored the BTB/POZ and ankyrin repeat domains (Backer et al. 2015). Phylogenetic analysis divided AtNPRI-like gene family into three functionally distinct clades (Peraza-Echeverria et al. 2012; Zhang et al. 2006). In the first clade, AtNPRI and AtNPR 2 were involved in a positive regulator of SAR (Cao et al. 1997). In the second clade, $A t N P R 3$ and $A t N P R 4$ took part in negative SAR regulation (Liu et al. 2004; Zhang et al. 2006). In the third clade, AtBOP1 and AtBOP2 were involved in the organ symmetry and determinacy during the leaf morphogenesis (Hepworth et al. 2005; McKim et al. 2008). The phylogenetic analysis indicated the functional characteristics of the NPRI-like gene family, providing valuable resources for further study.

$N P R 1$-like genes play an important role in resistance disease, growth, and development of plant tissues and organs in many plants, for examples, tomato, strawberry, avocado, banana (Backer et al. 2015; Endah et al. 2008; Li et al. 2019; Wang et al. 2018). However, NPR1-like gene family was still unidentified and uncharacterized in Chinese pear. In this study, it was the first report about discovering and identifying the NPRI-like gene family in Chinese pear genome. Also, $P b r N P R 1$-like genes were analyzed the phylogeny, gene structure, conserved motif, ciselements, chromosomal location, and tissue-specific expression of $P$. bretschneideri Reld. Using qPCR technology, we indicated how NPR1-like genes response after SA treatment and $A$.

alternata infection and expression changes within 24 hours. This study provided a valuable foundation for further functional analysis of the PbrNPR 1 -like genes and pear genetic improvement.

\section{Materials \& Methods}

\section{Plant Materials and Treatment}


120 The three-year-old P. bretschneideri Rehd (cv.Yali) trees were cultivated in the greenhouse (16 h $121 / 8 \mathrm{~h} \mathrm{light} /$ dark, $24 / 20^{\circ} \mathrm{C}$ day/night and relative humidity of $25 \%$ ) in the experimental farm of 122 Hebei Agricultural University, Baoding, Hebei, China. Yali leaves were collected at $0 \mathrm{~h}, 1 \mathrm{~h}, 3 \mathrm{~h}$, $1236 \mathrm{~h}, 12 \mathrm{~h}, 24 \mathrm{~h}, 48 \mathrm{~h}$, and $72 \mathrm{~h}$ after $0.2 \mathrm{mM} \mathrm{SA}$ treatment. Samples from different tissue of

124

125

126

127

128

129

130

131

132

133

134

135

136

137

138

139

140

141

142

143

144

145

146

147

148

149

150

151

152

153

154

155

156

157

158 seven-year-old Yali were gained at different stages of development. Flower buds were sampled 1 day before flowering, and then flower, young leaves, young stem, young fruit, immature leaves, old stem, mature fruits and seed were collected at 1, 15,15, 29,115, 115, 159 and 159 days after flowering (DAF) in 2018, respectively. The samples were taken at eight time points throughout the day, 9:00 am, 12:00 am, 15:00 pm, 18:00 pm, 21:00 pm, 24:00 am, 3:00 am and 6:00 am. The $A$. alternata was isolated from the diseased leaves, and grew at $28^{\circ} \mathrm{C}$ incubator.

\section{Identification of PbrNPR1-like gene family member}

The genome of P.bretschneideri Rehd (cv. Dangshan Suli) was downloaded from the Genome Database for Rosaceae (GDR) (http://www.rosaceae.org/) to identify the genome-wide NPRIlike gene family in Chinese pear. Using hmmsearch tool in the HMMER (v3.0) software package, we detected the PbrNPR1-like genes in the Protein database. HMMER file according to the ankyrin domain (PF00023) and BTB/POZ domain (PF00651) was downloaded from the Pfam protein database (http://pfam.xfam.org/). The CDS length, protein size, isoelectric point (IP), molecular weight (MW) of the deduced PbrNPRl-like genes were analyzed using the ExPASy website (https://web.expasy.org/compute_pi/).

\section{Sequence alignment and phylogenetic analysis}

The NPR1-like proteins sequences of rice, arabidopsis, grape, apple, pear, and other plants were obtained from NCBI and Ensamble plants (http://plants.ensembl.org/index.html) to perform alignments by using Clustal W 2.0 (Larkin et al. 2007). The phylogenetic tree was constructed through the neighbor-joining (NJ) method with the bootstrap value 1000 replicates in the MEGA 6.0 software (Tamura et al. 2013).

\section{Sequence analysis of PbrNPR1-like gene family}

The individual PbrNPR1-like genes structure was displayed using Gene Structure Display Server 2.0 software (GSDS, http://gsds.cbi.pku.edu.cn/) the exon/intron structure of based on the alignments of NPRI-like genes CDS and their genomic sequences in Chinese pear and Arabidopsis (Hu et al. 2015). The MEME software program (http://meme-suite.org/) was used to analyze the NPR1-like protein motif with the following parameters: 1) the width of optimum motif 6-200; 2) the maximum number of motif 10 (Bailey et al. 2009). The domain was analyzed by using a web CD-search tool (https://www.ncbi.nlm.nih.gov/Structure/bwrpsb/bwrpsb.cgi) (Lu et al. 2020).

\section{Cis-elements in the Promoters of PbrNPR1-like Genes}


159 We analyzed the promoter region up to 2000 bp except the Pbrgene12425 gene inclding 1300

160

161

162

163

164

165

166

167

168

169

170

171

172

173

174

175

176

177

178

179

180

181

182

183

184

185

186

187

188

189

190

191

192

193

194

195

196

197

198

bp. The promoter sequences of the PbrNPR1-like genes were analyzed using PlantCARE databases (http://bioinformatics.psb.ugent.be/webtools/plantcare/html/).

\section{Real-Time Quantitative PCR (qPCR)}

The total RNA of samples was extracted using the plant RNA extraction kit (Takara, Dalian, China). First-stand cDNA was synthesized through the FastQuant RT Kit with gDNase (TianGen, Beijing, China). The qPCR was performed using the Top Green qPCR SuperMix Kit (TranGen, Beijing, China). Each $20.0 \mu \mathrm{l}$ reaction mixture included $10.0 \mu \mathrm{l}$ SYBR supermix, 2.0 $\mu \mathrm{l}$ cDNA template, $0.4 \mu \mathrm{l}$ forward and reverse primer $(10.0 \mu \mathrm{M})$ and $7.2 \mu \mathrm{ddH}_{2} \mathrm{O}$.

LightCycler ${ }^{\circledR} 96$ system (Roche, Germany) was used for qPCR using the following PCR parameters: pre-denaturation $94^{\circ} \mathrm{C}$ for $30 \mathrm{~s}$, followed by 42 cycles of $94^{\circ} \mathrm{C}$ denaturation for $5 \mathrm{~s}$, $55^{\circ} \mathrm{C}$ annealing for $15 \mathrm{~s}, 72^{\circ} \mathrm{C}$ extension for $10 \mathrm{~s}$, melting-curve analysis was done by $95^{\circ} \mathrm{C}$ for $15 \mathrm{~s}$, and then a constant increase from 58 to $95^{\circ} \mathrm{C}$ at a $2 \%$ ramp rate. All gene-specific primers for $\operatorname{PbrNPR} 1$-like gene family were designed by using DNAMAN 8.0 software except Pbrgene33340, and listed in Supplementary file 1- Table S2 except Pbrgene33340 and three technical replicates for each biological replicate were performed. The relative expression of the PbrNPR1-like genes was calculated using the $2^{-\Delta \Delta \mathrm{CT}}$ method (Livak \& Schmittgen 2001), and three technical replicates and three biological replicate were applied.

\section{Statistical Analysis}

The results of analysis the variance (ANOVA) and duncan multiple comparison were analyzed using SPSS software (SPSS version 17.0, Chicago, IL, USA). Data was presented in graphs through OriginPro 9.1.0 software (Microcal Software, Inc., Northampton, MA, USA).

\section{Results}

\section{Identification of PbrNPR1-like genes}

After removing the redundant entries, nine PbrNPR1-like genes with typical BTB/POZ and ankryin domain were identified to unevenly distribute on the chromosomes. Among the nine PbrNPR1-like genes, three PbrNPR1-like genes were detected on chromosomes 5, the other 6 genes were identified on chromosome 2, 5, 9,10,14, and 17, respectively. The characteristics of PbrNPR 1-like genes were listed in Table 1, including the chromosomal location, intron number, coding sequence (CDS) length, amino acid length, protein molecular weight (MW) and isoelectric point (PI). The lengths of the open reading frame (ORF) of PbrNPRI-like genes range from 1494 bp (Pbrgene 40077/34018) to 1770 bp (Pbrgene8341). The length of the PbrNPR1like proteins range from 498 amino acids (Pbrgene40077/34018) to 591 amino acids (Pbrgene8341). The molecular weight of these proteins range from $54.52 \mathrm{kDa}$ (Pbrgene40077/ 34018) to $66.25 \mathrm{kDa}$ (Pbrgene8341) and the PIs range from 5.7 (Pbrgene8896/33340) to 6.4 (Pbrgene8895). All genes contain 1 or 3 introns.

Peer] reviewing PDF | (2021:04:59817:2:0:NEW 8 Sep 2021) 
199 Phylogenetic and protein domain analysis of PbrNPR1-like gene family

200 The Phylogenetic analysis of family determines the origin of the evolutionary process of the 201 family, and the internal branch of the evolutionary tree reflects the distance of evolutionary 202 relationships among different genes. To explore the evolutionary relationship of PbrNPR1-like 203 gene family, NPR1-like protein sequences from 20 different monocotyledon and dicotyledon 204 plant species were used to construct the phylogenetic tree (Supplementary file 1-Table S1). 205 According to the phylogenetic tree, these NPR1-like proteins were divided into three clades: 206 clade I , II, III. As shown in Fig. 1, Pbrgene 12425 was classified into the cluster of AtNPR1 and AtNPR2 as the positive regulator of SAR. However, the Pbrgene6286, Pbrgene8895, Pbrgene8896, Pbrgene2529, Pbrgene8341 and Pbrgene33340 involved in the clade II of AtNPR3 and AtNPR4 negative SAR regulation (Zhang et al. 2006). The other two protein Pbrgene34018 and Pbrgene 40077 were classified into the clade III along with AtBOP1 and AtBOP2 to participated in the organ determinacy and symmetry (Hepworth et al. 2005; McKim et al. 2008).

\section{Gene structure, motif composition, and domain of NPR1-like gene family}

The classification of PbrNPR1-like genes was presented in Fig. 2B, in which clade I and II both contained four exons and three introns, and the Clade III (Pbrgene34018 and Pbrgene40077) included two exons and one intron. The protein domain composition revealed that all NPR1-like proteins included an N-terminal BTB-POZ domain and the ankyrin repeat domain located central region (Fig. 2C). The genes in Clade I and the Clade II both have NPR1-like-C terminal region which has been proved to be an important part in NPR1-like gene family (Boyle et al. 2009; Rochon et al. 2006). The NPR1-like-C terminal protein size usually existed in the eukaryotes with the size between 251 and 588 amino acids. The $\mathrm{C}$-terminal region included a nuclear localization signal (NLS), a NIMINTERACTING (NIMIN) 1/2 protein binding sites, and a conservative penta-amino acid motif (LENRV) (Liu et al. 2019). The presumed domain DUF3420 was found in eukaryotes with the length about 50 amino acids which was functionally uncharacterized. The Pbrgene2529 did not have the DUF3420 domain.

Ten conserved motifs were identified in the PbrNPR1-like gene family (Supplementary file 1Fig. S1, Table S3). As shown in Fig. 2D, Clade I and Clade II harbored motif 1-10 and Clade III featured motifs $1,2,3,5,6,7,9$, and 10 . The same conserved motif ingredients in each clade supported the polygenetic classification in the PbrNPR1-like gene family.

Multiple sequence alignments were executed to examine the conservation of residues, domains, and motifs in PbrNPR1-like and known-function AtNPR1-like (AtNPR1 to AtNPR6) (Fig. 2E). We found that npr1-2 (Cys150Tyr), nim1-2 (His300Tyr), and npr1-1 (His334Tyr) mutations in AtNPR1 were completely conserved in all PbrNPR1-like proteins. Nim1-4 (Arg432Lys) in AtNPR1 and npr4-4D (Arg419Gln) in AtNPR4 mutant sites were conserved in Clade I and Clade II. C82, C216, and C156 cysteine residues in AtNPR1 participated in its oligomer-monomer transition were highly conservation in PbrNPR1-like proteins. The Arg432 residues in AtNPR1, Arg428 in AtNPR3, and Arg419 in AtNPR4 required for their expression of SA were conserved in Clade I and Clade II in PbrNPR1-like protein. 
239

240

241

242

243

244

245

246

247

248

249

250

251

252

253

254

255

256

257

258

259

260

261

262

263

264

265

266

267

268

269

270

271

272

273

274

275

276

277

\section{Cis-elements in the promoter regions of PbrNPR1-like genes}

In this study, about 2000 bp promoter region sequence was identified in the PbrNPRl-like gene family except for the Pbrgene12425 with $1300 \mathrm{bp}$. The cis-elements were divided into three aspects through the PlantCARE database, including plant growth and development, stress responses, and hormone responses (Supplementary file 1- Table S4). In the first aspect, MRE, Gbox, GTI motif, GATA motif, I-box, AE-box, ATCT-motif, TCT motif, TCCC motif and Box 4 for light responsiveness, circadian for the circadian control, $\mathrm{O}_{2}$-site for zein metabolism regulation (Fig. 3) (Abdullah et al. 2018). In the second aspect, included a range of stress-related elements, such as, STRE and TC-rich repeats involved in stress responses; MBS, MYB, MYC involved drought inducibility, ARE involved in anaerobic induction, W box was a SA-induced WRKY transcription factors, The activation sequence (as-1) element took part in the transcription activation several SA-regulated PR genes (Hernandez-Garcia \& Finer 2014; Yamaguchi-Shinozaki \& Shinozaki 2006). WUN-motif related to a wound-responsive element (Ni et al. 1996), LTR for low-temperature responsiveness (Xiang et al. 2007). The third aspect, we detected the ABRE related to ABA was the most ordinary motif (Kim et al., 2011), CGTAC motif and TGACG motif for MeJA responsiveness, GARE-motif, P-box, and TATC box for gibberellin responsiveness elements, TCA-element for SA responsiveness, the TGA-element for auxin-response (Abdullah et al. 2018). These results indicated that PbrNPRl-like genes family have the advantages for enhancing abiotic stress responses and hormones responsiveness and may respond to abiotic stress and hormones.

\section{Expression patterns for the PbrNPR1-like genes in different plant tissues}

To obtain a first glance at the functions of PbrNPRl-like genes during different developmental stages of pear, the transcript accumulation levels were observed in 10 tissues, including the leaves (April, July), stems (April, July), flowers (4 April), flowers buds (8 April), fruits (May, September), seed (September) and bud ( 8 March) during the budding and reproductive stages.

The result revealed that $P b r N P R 1$-like genes were constitutively expressed in different tissue, and the expression level were varied (Fig. 4). The expressions levels of PbrNPR1-like genes in young fruits, mature leaves and mature stem were higher than that in mature fruits, young leaves and young stem, except for Pbrgene6268/8896/12425. Among the nine genes, the expression level of Pbrgene6286 gene was relatively stable in 10 tissues/organs, Pbrgene12425 gene was moderately expressed in leaves, Pbrgene 8895 was highly expressed in leaf, stem, seed and bud tissue, Pbrgene8341/8896 were relatively highly expression in leaves, Pbrgene34018/40077 were highly expressed in flower, bud and stem. The expression patterns of clade II in the ten tissues were very similar.

\section{Differential expression pattern of the PbrNPR1-like genes in response to SA} treatment 
278 The transcript levels of Pbrgene2529/6286/8341/8895/12425/34018/40077 were up-regulated 279 among that the Pbrgene 2529/8341/8895/12425 reached their highest expression levels at $12 \mathrm{~h}$ 280 with 2.29-fold, 2.72-fold, 6.86-fold, and 3.96-fold comparing to the $0 \mathrm{~h}$ samples, respectively, 281 and then slowly returned to the baseline levels after $12 \mathrm{~h}$ (Fig. 5). Comparing to the $0 \mathrm{~h}$ samples, 282 the expression levels of Pbrgene6286, Pbrgene34018, Pbrgene 40077 were the highest at $6 \mathrm{~h}$ 283 with 4.45 -fold, $3 \mathrm{~h}$ with 2.57 -fold, $3 \mathrm{~h}$ with 1.97 -fold, respectively. Whereas Pbrgene 8896 was 284 first down-regulated and then up-regulated at $24 \mathrm{~h}$. We observed the expression of the 285 Pbrgene6286/8341/12425 gene changed more than other PbrNPRI-like genes. In brief, the 286

287 expression patterns of $P b r N P R 1$-like genes were significantly affected by exogenous SA hormones.

288

289

290

291

292

293

294

295

296

297

298

299

300

301

302

303

304

305

306

307

308

309

310

311

312

313

314

315

316

317

\section{Expression analysis of PbrNPR1-like genes in response to A.alternata.}

To confirm the potential functions of the $P b r N P R l$-like gene family in response to biotic stress, the expression changes of the $P b r N P R I$-like genes were compared after challenge with A.alternata. All the PbrNNPR1-like genes can be induced by infected with A.alternata through varied expression patterns. The expression of Pbrgene6286/8895/12425/34018/40077 was upregulated and reached highest at $96 \mathrm{hpi}$ or $120 \mathrm{hpi}$, meanwhile these genes were reached a small peak at 12 hpi (Fig. 6). The expression of Pbrgene1 2425 was significantly higher than the other PbrNPR1-like genes at 96 hpi. The Pbrgene2529/8341/8896 was up-regulated and reached a peak at $12 \mathrm{hpi}$, and then dramatically decreased except Pbrgene 8896 was then up-regulated at 72 hpi.

\section{Differential expression profile of the PbrNPR1-like genes in the circadian rhythm} The circadian rhythm expression patterns of $P b r N P R 1$-like genes under long daytime were compared. The results showed that the expression of all the PbrNPR1-like genes had the phenomenon of circadian rhythm. Most of the nine genes had similar expressed patterns except for Pbrgene40077, which were expressed higher in the daytime than that in the night (Fig. 7). The expression of Pbrgene 40077 was the highest at 21:00 pm, and low in all other time. This circadian clock phenomenon was related to the function of the family members, the clade I, II involved in plant immune, and the clade III took part in the organ symmetry and determinacy during the leaf morphogenesis.

\section{Discussion}

Nine PbrNPR 1-like genes, Pbrgene12425/8895/8896/8341/6286/34018/2529/33340/40077 were identified in Chinese pear. PbrNPR1-like proteins have similar gene structures, domain, and conserved motifs and amino acid residues with NPRI-like sequences in Arabidopsis, suggesting that their orthologs probably display similar biological functions in Chinese pear, while the difference between the groups specified their function was diversified. Similar results had existed in Oryza sativa, Malus domestica, Populus trichocarpa (Shao et al. 2013; Yuan et al. 2007; Zhang et al. 2016). These findings indicated that the NPRI-like genes were highly 
318 conserved in numerous plant species. The result of phylogenetic tree suggested that potential

319

320

321

322

323

324

325

326

327

328

329

330

331

332

333

334

335

336

337

338

339

340

341

342

343

344

345

346

347

348

349

350

351

352

353

354

355

356

357

functional distinctions between the $P b r N P R 1$-like gene family were existed.

A large number of stress response elements were existed in promoter regions, such as ARE, LTR, MBS, MYB, MYC, STRE, TC-rich repeats, which suggested that PbrNPRl-like genes played an important role in the stress response process. In addition, G-box, I-box, and other optical response elements were found in the promoter regions, indicating that these genes may also be regulated by optical signals. Meanwhile, we detected the ABRE, CGTAC, TGACG, GARE, TATC box, P-box, TCA-element, and TGA-element in the nine genes, so we speculate these genes take part in the hormone-responsive and be induced by the GA, SA, ABA, auxin, and MeJA hormone (Zhao et al. 2020). All the results implied that the different PbrNPRl-like genes played a role in special environment at different times, and thus the patterns of PbrNPRI-like genes responses to hormones were complex.

To further state the possible functions of the NPRI-like gene family in the development and growth of P.bretschneideri Rehd, the transcription profiles of $P b r N P R 1$-like genes were studied through qRT-PCR in ten different tissues. The study displayed Pbrgene 12425 was expressed in all the tissues with the highest expression in mature leaves. Pbrgene6268 and Pbrgene 8896 was expressed more higher in flower bud than that in flower. The tissue- and stage-specific expression profile implied a specific function of the two genes in the early flower development (Shi et al. 2013). Pbrgene34018 and Pbrgene 40077 in clade III displayed specific expression in the leaves, stem, and flower (Fig. 4), implying that they may participated in the organ symmetry and determinacy during the leaf morphogenesis which was similar to the study on Persea Americana. The expression of PaNPR1, PaNPR2, and PaNPR4 were seen in all sorts of tissues (Backer et al. 2015). The expression of $P a N P R 3$ was expressed much higher in fruit and aerial tissues. The PaNPR5 was displayed in the roots. Therefore, database retrieval and functional prediction of $P b r N P R 1$-like genes in different tissues and various stages of development indicated that $P b r N P R 1$-like genes may play an important role in plant growth, and some PbrNPRI-like genes may have unique functions at specific stages of development.

The expression profile of the PbrNPRI-like genes showed obviously different after SA induction. The same result were obtained in Qinguan apple resistant disease cultivars, the $M d N P R 1 / 2 / 3 / 4 / 5 / 6 / 7$ were up-regulated at $6 \mathrm{~h}$, and $M d N P R 8$ were expressed to the highest level at $12 \mathrm{~h}$ (Zhang et al. 2016). The time of reaching peak expression is different in the various plants, the possible reason is that different defense system and the time of the infection and reaction varies. The expression of defense-related genes increased after 3 hours in the morning with SA inducing in the morning or evening under constant light for 3 hours while the expression of growth-related genes increased in the evening (Zhou et al. 2015). The NPRI-like genes would be induced after SA treatment and participated in the SA signal transduction which plays an important role in local defense and distal tissue of wide-spectrum SAR (Baldwin \& Meldau 2013).

Plant disease activated by the pathogens promote defense immunity to plant pathogens and the defense immunity is a difficult mechanism including the triggering of multiple immune

Peer) reviewing PDF | (2021:04:59817:2:0:NEW 8 Sep 2021) 
358

359

360

361

362

363

364

365

366

367

368

369

370

371

372

373

374

375

376

377

378

379

380

381

382

383

384

385

386

387

388

389

390

391

392

393

394

395

396

mechanisms reaction (Spoel \& Dong 2012). Several resistant genes and proteins associated with the pathogenesis have been isolated and they can be used to improve the plant defense against different diseases. The PR proteins play an important role in plant defense systems. NPR1 can interact with some members of the TGA family of bZIP transcriptions factors which combine with the as-1-like (TGACG) element in the PR gene promoter and take charge of PR gene expression (Zhang et al. 2003; Zhou et al. 2000). The results (Fig. 6) not only showed that there were significantly different in the expression of the family genes after infecting with A.alternata and some PbrNPRl-like genes were assciated with pear resistance to A.alternata in pear. PbrNPRl-like genes were pathogen-inducible and participated in the immune system of pear, enhancing the disease resistance.

Studies have found that the expression of NPRI has circadian rhythm in the A.thaliana (Zhou et al. 2015), which is balanced between the regulation of self-growth and immunity. The expression of $P b r N P R 1$-like gene family showed circadian rhythm in natural conditions during a day, indicating that the PbrNPRI-like genes may be conserved in plants circadian rhythm. The stomata on the surface of plant leaves opens in the morning, making it vulnerable to pathogen invasion, the plant activates a defense mechanism which makes it more resistant to disease in the morning than at night, the plant grows normally at night (Korneli et al. 2014; Zhang et al. 2013). This result verified the conclusion that SA mediated immune responses in the morning helped to avoid the conflicts between SA immune responses and growth-related activities that need to transport water at night (Zhou et al. 2015). This change in plant defense response may reflect an adaptation to change physical conditions during the day and the temperatures and humidity are generally more conducive to pathogen challenges in the morning (Karapetyan \& Dong 2018).

The functions of the NPRI-like genes maybe much more intricate in Chinese pear than A.thaliana. Overexpression of AtNPRI can improve the disease resistance of A.thaliana, while AtNPR3 and AtNPR4 have redundant functions and opposite functions to the AtNPRI (Ding et al. 2018). When the biotic and abiotic infection, SA will combine with its receptors (NPR1, NPR3, and NPR4) and induce the expression of PR protein, which leads to triggering immune responses. AtNPR2 was more similar to the AtNPR1 than the other NPR 1 orthologs and would play an important role in the SA perception and acted as an evolutive reservoir of the NPRI (Castello et al. 2018). In pear, it was speculated there were more homologs would interact with each other to regulate the balance PbrNPR1. The PbrNPR1 homologs can be regulated by transcribed to different transcripts or transcription factors.

This study provided evidence to assist the preliminary $P b r N P R 1$-like gene family information and functional annotation of the nine discovered $P b r N P R 1$-like genes from pear. Sequence structure, homology, and phylogenetic analysis suggested that seven PbrNPRI-like proteins might participate in the defense responses, the rest two genes were likely involved in tissue development. Hormone and expression in various tissues provided a support for this and allow future research to learn much more about the possible role of the PbrNPRI-like genes in SAR in pear. The future efforts will be focused on the localization and the intracellular interactions of 
397

398

399

400

401

402

403

404

405

406

407

408

409

410

411

412

413

414

415

416

417

418

419

420

421

422

423

424

425

426

427

428

429

430

431

432

433

434

435

436

437

438

439

440

441

442

defense-related $\mathrm{PbrNPR}$-like proteins as well as the role of overexpressing $\mathrm{PbrNPR} 1$-like genes in the nprl mutant and wild-type Arabidopsis.

\section{Conclusions}

Based on genomic data of P.bretschneideri Reld, we identified nine PbrNPRl-like genes. We conducted phylogenetic analyses, as well as conserved domain, conversed motif, promoter and expression profiling of the PbrNPR1-like gene family under SA and A.alternata. According to the structural and phylogenetic characteristics of the PbrNPR1-like protein sequences, they were divided into three clades. Most of the genes were responsive to SA and A.alternata. The expression of all the PbrNPR1-like genes had the phenomenon of circadian rhythm which most genes were expressed higher in the daytime than night except for the Pbrgene40077. These findings provide a solid insight for understanding the functions and evolution of PbrNPRl-like genes in Chinese pear. Future studies can be performed for gene function for the mechanism of resistance disease in Yali.

\section{References}

Abdullah M, Cao Y, Cheng X, Meng D, Chen Y, Shakoor A, Gao J, and Cai Y. 2018. The Sucrose Synthase Gene Family in Chinese Pear (Pyrus bretschneideri Rehd.): Structure, Expression, and Evolution. Molecules 23. 10.3390/molecules23051144

An C, and Mou Z. 2011. Salicylic acid and its function in plant immunity. J Integr Plant Biol 53:412-428. 10.1111/j.1744-7909.2011.01043.x

Attaran E, and He SY. 2012. The long-sought-after salicylic acid receptors. Molecular Plant 5:971-973. $10.1093 / \mathrm{mp} / \mathrm{sss} 086$

Backer R, Mahomed W, Reeksting BJ, Engelbrecht J, Ibarra-Laclette E, and van den Berg N. 2015. Phylogenetic and expression analysis of the NPR1-like gene family from Persea americana (Mill.). Frontiers in Plant Science 6:300-314. 10.3389/fpls.2015.00300

Bailey TL, Boden M, Buske FA, Frith M, Grant CE, Clementi L, Ren J, Li WW, and Noble WS. 2009. MEME suite: tools for motif discovery and searching. Nucleic Acids Research 37:W202-208. 10.1093/nar/gkp335

Baldwin IT, and Meldau S. 2013. Just in time: circadian defense patterns and the optimal defense hypothesis. Plant Signaling \& Behavior 8:e24410. 10.4161/psb.24410

Boyle P, Le Su E, Rochon A, Shearer HL, Murmu J, Chu JY, Fobert PR, and Despres C. 2009. The BTB/POZ domain of the Arabidopsis disease resistance protein NPR1 interacts with the repression domain of TGA2 to negate its function. The Plant Cell 21:3700-3713. 10.1105/tpc.109.069971

Cao H, Glazebrook J, Clarke JD, Volko S, and Dong X. 1997. The Arabidopsis NPR1 gene that controls systemic acquired resistance encodes a novel protein containing ankyrin repeats. Cell 88:57-63. 10.1016/s0092-8674(00)81858-9

Cao H, Li X, and Dong X. 1998. Generation of broad-spectrum disease resistance by overexpression of an essential regulatory gene in systemic acquired resistance. Proceedings of the National Academy of the United States of America 95:6531-6536. 10.1073/pnas.95.11.6531

Castello MJ, Medina-Puche L, Lamilla J, and Tornero P. 2018. NPR1 paralogs of Arabidopsis and their role in salicylic acid perception. PLoS One 13:e0209835. 10.1371/journal.pone.0209835 
443

444

445

446

447

448

449

450

451

452

453

454

455

456

457

458

459

460

461

462

463

464

465

466

467

468

469

470

471

472

473

474

475

476

477

478

479

480

481

482

483

484

485

486

487

488

489

490

491

Chen J, Mohan R, Zhang Y, Li M, Chen H, Palmer IA, Chang M, Qi G, Spoel SH, Mengiste T, Wang D, Liu F, and Fu ZQ. 2019. NPR1 promotes its own and target gene expression in plant defense by recruiting CDK8. Plant Physiology 181:289-304. 10.1104/pp.19.00124

Chen XK, Zhang JY, Zhang Z, Du XL, Du BB, and Qu SC. 2012. Overexpressing MhNPR1 in transgenic Fuji apples enhances resistance to apple powdery mildew. Molecular Biology Reports 39:8083-8089. 10.1007/s11033-012-1655-3

Ding Y, Sun T, Ao K, Peng Y, Zhang Y, Li X, and Zhang Y. 2018. Opposite roles of Salicylic Acid receptors NPR1 and NPR3/NPR4 in transcriptional regulation of plant immunity. Cell 173:1454-1467 e1415. 10.1016/j.cell.2018.03.044

Dodd AN, Salathia N, Hall A, Kevei E, Toth R, Nagy F, Hibberd JM, Millar AJ, and Webb AAR. 2005. Plant circadian clocks increase photosynthesis, growth, survival, and competitive advantage. Science 309:630-633. 10.1126/science.1115581

Dong X. 2004. NPR1, all things considered. Curr Opin Plant Biol 7:547-552. 10.1016/j.pbi.2004.07.005

Endah R, Beyene G, Kiggundu A, van den Berg N, Schluter U, Kunert K, and Chikwamba R. 2008. Elicitor and Fusarium-induced expression of NPR1-like genes in banana. Plant Physiology Biochemistry 46:1007-1014. 10.1016/j.plaphy.2008.06.007

Fu ZQ, Yan S, Saleh A, Wang W, Ruble J, Oka N, Mohan R, Spoel SH, Tada Y, Zheng N, and Dong X. 2012. NPR3 and NPR4 are receptors for the immune signal salicylic acid in plants. Nature 486:228-232. 10.1038/nature11162

Hepworth SR, Zhang Y, McKim S, Li X, and Haughn GW. 2005. Blade-On-Petiole-dependent signaling controls leaf and floral patterning in Arabidopsis. The Plant Cell 17:1434-1448. 10.1105/tpc. 104.030536

Hernandez-Garcia CM, and Finer JJ. 2014. Identification and validation of promoters and cisacting regulatory elements. Plant ence 217-218:109-119.

Hu B, Jin J, Guo AY, Zhang H, Luo J, and Gao G. 2015. GSDS 2.0: an upgraded gene feature visualization server. Bioinformatics 31:1296-1297. 10.1093/bioinformatics/btu817

Karapetyan S, and Dong X. 2018. Redox and the circadian clock in plant immunity: a balancing act. Free Radical Biology and Medicine 119:56-61. 10.1016/j.freeradbiomed.2017.12.024

Kinkema M, Fan WH, and Dong XN. 2000. Nuclear localization of NPR1 is required for activation of PR gene expression. The Plant Cell 12:2339-2350. DOI 10.1105/tpc.12.12.2339

Korneli C, Danisman S, and Staiger D. 2014. Differential control of pre-invasive and postinvasive antibacterial defense by the Arabidopsis circadian clock. Plant Cell Physiol 55:1613-1622. 10.1093/pcp/pcu092

Larkin MA, Blackshields G, Brown NP, Chenna R, McGettigan PA, McWilliam H, Valentin F, Wallace IM, Wilm A, Lopez R, Thompson JD, Gibson TJ, and Higgins DG. 2007. Clustal $W$ and Clustal $X$ version 2.0. Bioinformatics 23:2947-2948. 10.1093/bioinformatics/btm404

Lawton K, Weymann K, Friedrich L, Vernooij B, Uknes S, and Ryals J. 1995. Systemic acquired resistance in Arabidopsis requires salicylic acid but not ethylene. Mol Plant Microbe Interact 8:863-870. 10.1094/mpmi-8-0863

Li R, Liu C, Zhao R, Wang L, Chen L, Yu W, Zhang S, Sheng J, and Shen L. 2019. CRISPR/Cas9-Mediated SINPR1 mutagenesis reduces tomato plant drought tolerance. BMC Plant Biology 19:38. 10.1186/s12870-018-1627-4

Liu G, Holub EB, Alonso JM, Ecker JR, and Fobert PR. 2004. An Arabidopsis NPR1-like gene, NPR4, is required for disease resistance. The Plant Journal 41:304-318. 10.1111/j.1365313X.2004.02296.X

Peer] reviewing PDF | (2021:04:59817:2:0:NEW 8 Sep 2021) 
492

493

494

495

496

497

498

499

500

501

502

503

504

505

506

507

508

509

510

511

512

513

514

515

516

517

518

519

520

521

522

523

524

525

526

527

528

529

530

531

532

533

534

535

536

537

538

539

540

Liu X, Liu Z, Niu X, Xu Q, and Yang L. 2019. Genome-Wide identification and analysis of the NPR1-Like gene family in bread wheat and its relatives. International Journal of Molecular Science 20. 10.3390/ijms20235974

Livak KJ, and Schmittgen TD. 2001. Analysis of relative gene expression data using real-time quantitative PCR and the 2(-Delta Delta C(T)) Method. Methods 25:402-408. 10.1006/meth.2001.1262

Lu S, Wang J, Chitsaz F, Derbyshire MK, Geer RC, Gonzales NR, Gwadz M, Hurwitz DI, Marchler GH, Song JS, Thanki N, Yamashita RA, Yang M, Zhang D, Zheng C, Lanczycki CJ, and Marchler-Bauer A. 2020. CDD/SPARCLE: the conserved domain database in 2020. Nucleic Acids Reseach 48:D265-D268. 10.1093/nar/gkz991

McKim SM, Stenvik GE, Butenko MA, Kristiansen W, Cho SK, Hepworth SR, Aalen RB, and Haughn GW. 2008. The Blade-On-Petiole genes are essential for abscission zone formation in Arabidopsis. Development 135:1537-1546. 10.1242/dev.012807

Mou Z, Fan W, and Dong X. 2003. Inducers of plant systemic acquired resistance regulate NPR1 function through redox changes. Cell 113:935-944. 10.1016/s00928674(03)00429-x

$\mathrm{Ni}$, Min, Decai, CuiStanton, B., and Gelvin. 1996. Sequence-specific interactions of woundinducible nuclear factors with mannopine synthase 2' promoter wound-responsive elements. Plant Molecular Biology 30:77-96.

Nie P, Li X, Wang S, Guo J, Zhao H, and Niu D. 2017. Induced systemic resistance against botrytis cinerea by bacillus cereus AR156 through a JA/ET- and NPR1-dependent signaling pathway and activates PAMP-triggered immunity in Arabidopsis. Frontiers in Plant Science 8. 10.3389/fpls.2017.00238

Peraza-Echeverria S, Santamaría JM, Fuentes G, de los Ángeles Menéndez-Cerón M, VallejoReyna MÁ, and Herrera-Valencia VA. 2012. The NPR1 family of transcription cofactors in papaya: insights into its structure, phylogeny and expression. Genes \& Genomics 34:379-390. 10.1007/s13258-011-0218-7

Pieterse CM, Leon-Reyes A, Van der Ent S, and Van Wees SC. 2009. Networking by smallmolecule hormones in plant immunity. Nat Chem Biol 5:308-316. 10.1038/nchembio.164

Prodhan Y, Issak M, Munemasa S, Nakamura Y, and Murata Y. 2020. Salicylic acid receptor NPR1 is involved in guard cell chitosan signaling. Bioscience Biotechnology Biochemistry 84:963-969. 10.1080/09168451.2020.1718485

Robert-Seilaniantz A, Grant M, and Jones JD. 2011. Hormone crosstalk in plant disease and defense: more than just jasmonate-salicylate antagonism. Annu Rev Phytopathol 49:317-343. 10.1146/annurev-phyto-073009-114447

Rochon A, Boyle P, Wignes T, Fobert PR, and Despres C. 2006. The coactivator function of Arabidopsis NPR1 requires the core of its BTB/POZ domain and the oxidation of Cterminal cysteines. Plant Cell 18:3670-3685. 10.1105/tpc.106.046953

Shao Y, Zhang H, He H, Cheng B, and Xiang Y. 2013. Molecular cloning and characterization of orthologues of NPR1 gene from poplar. Journal of Phytopathology 161:35-42. 10.1111/jph.12002

Shi Z, Maximova S, Liu Y, Verica J, and Guiltinan MJ. 2013. The salicylic acid receptor NPR3 is a negative regulator of the transcriptional defense response during early flower development in Arabidopsis. Mol Plant 6:802-816. 10.1093/mp/sss091

Somers DE, Webb AA, Pearson M, and Kay SA. 1998. The short-period mutant, toc1-1, alters circadian clock regulation of multiple outputs throughout development in Arabidopsis thaliana. Development 125:485-494.

Spoel SH, and Dong X. 2012. How do plants achieve immunity? Defence without specialized immune cells. Nature Reviews Immunology 12:89-100. 10.1038/nri3141

Peer) reviewing PDF | (2021:04:59817:2:0:NEW 8 Sep 2021) 
541

542

543

544

545

546

547

548

549

550

551

552

553

554

555

556

557

558

559

560

561

562

563

564

565

566

567

568

569

570

571

572

573

574

575

576

577

578

579

580

581

582

583

584

585

586

587

588

589

590

Tamura K, Stecher G, Peterson D, Filipski A, and Kumar S. 2013. MEGA6: Molecular Evolutionary Genetics Analysis Version 6.0. Molecular Biology and Evolution 30:27252729. 10.1093/molbev/mst197

Wang Z, Shu LJ, Liao JY, Lin NC, and Chung C-L. 2018. Identification of a strawberry NPR-like gene involved in negative regulation of the salicylic acid-mediated defense pathway. PLoS One 13. 10.1371/journal.pone.0205790

Wu Y, Zhang D, Chu JY, Boyle P, Wang Y, Brindle ID, De Luca V, and Despres C. 2012. The Arabidopsis NPR1 protein is a receptor for the plant defense hormone salicylic acid. Cell Reports 1:639-647. 10.1016/j.celrep.2012.05.008

Xiang Y, Huang Y, and Xiong L. 2007. Characterization of stress-responsive CIPK genes in rice for stress tolerance improvement. Plant Physiol 144:1416-1428. 10.1104/pp.107.101295

Xu YQ, Wang H, Qin RL, Fang LJ, Liu Z, Yuan SS, Gai YP, and Ji XL. 2019. Characterization of NPR1 and NPR4 genes from mulberry (Morus multicaulis) and their roles in development and stress resistance. Physiologia Plantarum 167:302-316. $10.1111 / \mathrm{ppl} .12889$

Yamaguchi-Shinozaki K, and Shinozaki K. 2006. TRANSCRIPTIONAL REGULATORY NETWORKS IN CELLULAR RESPONSES AND TOLERANCE TO DEHYDRATION AND COLD STRESSES. Annual Review of Plant Biology 57:781-803.

Yuan Y, Zhong S, Li Q, Zhu Z, Lou Y, Wang L, Wang J, Wang M, Li Q, Yang D, and He Z. 2007. Functional analysis of rice NPR1-like genes reveals that OsNPR1/NH1 is the rice orthologue conferring disease resistance with enhanced herbivore susceptibility. Plant Biotechnology Journal 5:313-324. 10.1111/j.1467-7652.2007.00243.x

Zhang C, Xie Q, Anderson R, Ng G, Seitz N, Peterson T, McClung C, McDowell J, Kong D, Kwak J, and Lu H. 2013. Crosstalk between the circadian clock and innate immunity in Arabidopsis. PLoS Pathogens 9:e1003370. 10.1371/journal.ppat.1003370

Zhang J, Jiao P, Zhang C, Tong X, Wei Q, and Xu L. 2016. Apple NPR1 homologs and their alternative splicing forms may contribute to SA and disease responses. Tree Genetics \& Genomes 12. 10.1007/s11295-016-1050-7

Zhang J, Ren Z, Zhou Y, Ma Z, Ma Y, Hou D, Xu Z, and Huang X. 2019. NPR1 and redox rhythmx: connections, between circadian clock and plant immunity. International Journal of Molecular Science 20. 10.3390/ijms20051211

Zhang Y, Cheng YT, Qu N, Zhao Q, Bi D, and Li X. 2006. Negative regulation of defense responses in Arabidopsis by two NPR1 paralogs. The Plant Journal 48:647-656. 10.1111/j.1365-313X.2006.02903.x

Zhang Y, Tessaro MJ, Lassner M, and Li X. 2003. Knockout analysis of Arabidopsis transcription factors TGA2, TGA5, and TGA6 reveals their redundant and essential roles in systemic acquired resistance. The Plant Cell 15:2647-2653. 10.1105/tpc.014894

Zhao S, Wei Y, Pang H, Xu J, Li Y, Zhang H, Zhang J, and Zhang Y. 2020. Genome-wide identification of the PEBP genes in pears and the putative role of PbFT in flower bud differentiation. PeerJ 8:e8928. 10.7717/peerj.8928

Zhou JM, Trifa Y, Silva H, Pontier D, and Klessig DF. 2000. NPR1 differentially interacts with members of the TGA/OBF family of transcription factors that bind an element of the PR1 gene required for induction by salicylic acid. Molecular Plant Microbe Interactions 13:191-202. 10.1128/AEM.71.5.2558-2563.2005.

Zhou M, Wang W, Karapetyan S, Mwimba M, Marques J, Buchler NE, and Dong X. 2015. Redox rhythm reinforces the circadian clock to gate immune response. Nature 523:472476. 10.1038/nature14449

Peer) reviewing PDF | (2021:04:59817:2:0:NEW 8 Sep 2021) 
Table $\mathbf{1}$ (on next page)

Feature of NPR1-like genes identified in pear 


\begin{tabular}{|c|c|c|c|c|c|c|c|c|}
\hline Gene ID & Chromosome & Start & End & Intron Num & CDS(bp) & $\operatorname{Size}(a a)$ & $\mathrm{MW}(\mathrm{Da})$ & $\mathrm{pI}$ \\
\hline Pbrgene12425 & $\mathrm{Chr} 2$ & 3805908 & 3808655 & 3 & 1770 & 590 & 65439.61 & 6.17 \\
\hline Pbrgene 33340 & Chr5 & 22753538 & 22756434 & 3 & 1740 & 580 & 64113.4 & 5.7 \\
\hline Pbrgene8895 & Chr5 & 19907569 & 19910942 & 3 & 1758 & 586 & 65134.72 & 6.4 \\
\hline Pbrgene 8896 & Chr5 & 19914984 & 19917980 & 3 & 1740 & 580 & 64171.48 & 5.7 \\
\hline Pbrgene 8341 & Chr9 & 12770720 & 12774971 & 3 & 1773 & 591 & 66253.22 & 5.87 \\
\hline Pbrgene 6286 & Chr.10 & 20497900 & 20501536 & 3 & 1758 & 586 & 65005.3 & 5.76 \\
\hline Pbrgene 34018 & Chr14 & 9853384 & 9856337 & 1 & 1494 & 498 & 54516.7 & 6.21 \\
\hline Pbrgene2529 & Chr17 & 12796106 & 12799482 & 3 & 1749 & 583 & 65379.36 & 5.95 \\
\hline Pbrgene 40077 & Scaffald 008989209.1 & 75582 & 72762 & 1 & 1494 & 498 & 54516.7 & 6.21 \\
\hline
\end{tabular}




\section{Figure 1}

Phylogenetic analysis of the NPR1-like proteins from P.bretschneideri Rehd and other species.

A phylogenetic tree of nine NPR1-like proteins from P.bretschneideri Rehd as well as other monocotyledons and dicotyledons plant species. The red star remark the NPR1-like protein in P.bretschneider Rehd. The blue circle remark the NPR1-like protein in A.thaliana. The tree was reconstructed in MEGA software v6.0 using the Neighbor-joining (NJ) tree. A thousand bootstrap replicates were performed to assess the tree reliability. 


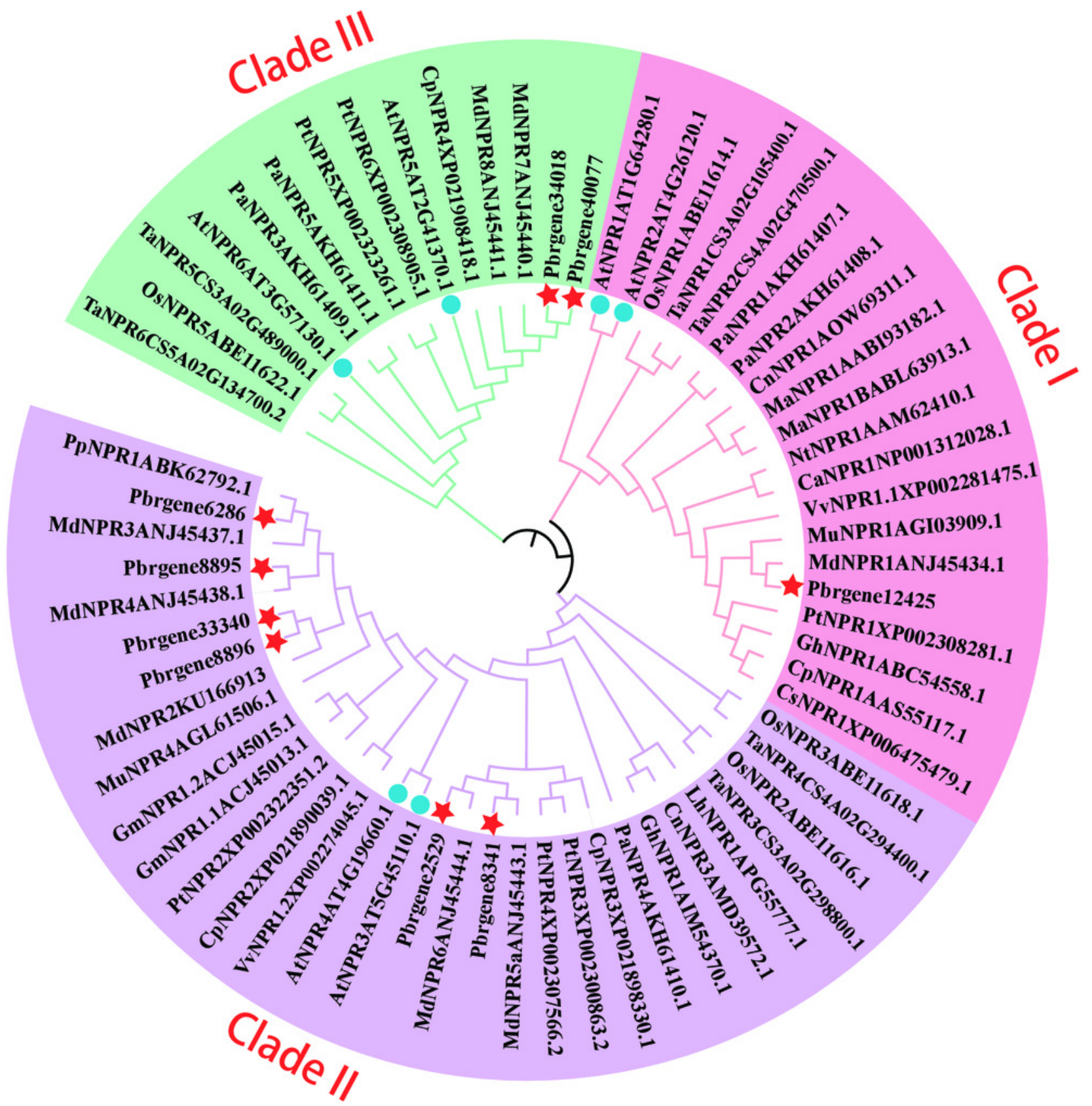




\section{Figure 2}

Gene structure and protein sequence comparison of PbrNPR1-like genes with AtNPR1like sequences.

(A) Phylogenetic relationship of NPR1-like genes in A.thaliana and P.bretschneideri Reld. (B) Exons, introns, and UTRs are showed by yellow boxes, grey lines, and blue boxes, respectively. (C) Conserved domain of the BTB/POZ, Ank, and NPR1-like C-terminal region in A.thaliana and P.bretschneideri Rehd. NPR1-like proteins. (D) The motifs were identified by MEME database with the protein sequence. (E) A multiple alignment of amino acid sequences of P.bretschneideri Rehd NPR1-like proteins (PbrNPR1 to PbrNPR6) and A.thaliana NPR1-like proteins (AtNPR1 to AtNPR6). npr1-1, npr1-2, nim1-2, and nim1-4 in AtNPR1 mutation sites, and highly conserved cysteines residues (C82, C216, and C156 in AtNPR1) are indicated with black arrows. The conserved domains, BTB/POZ and ANK, and some important motifs, putative hinge region (LENRV), EAR-like repression motif (VDLNETP), NIMIN-binding region, and nuclear localization signal (NLS), are indicated with solid lines. 

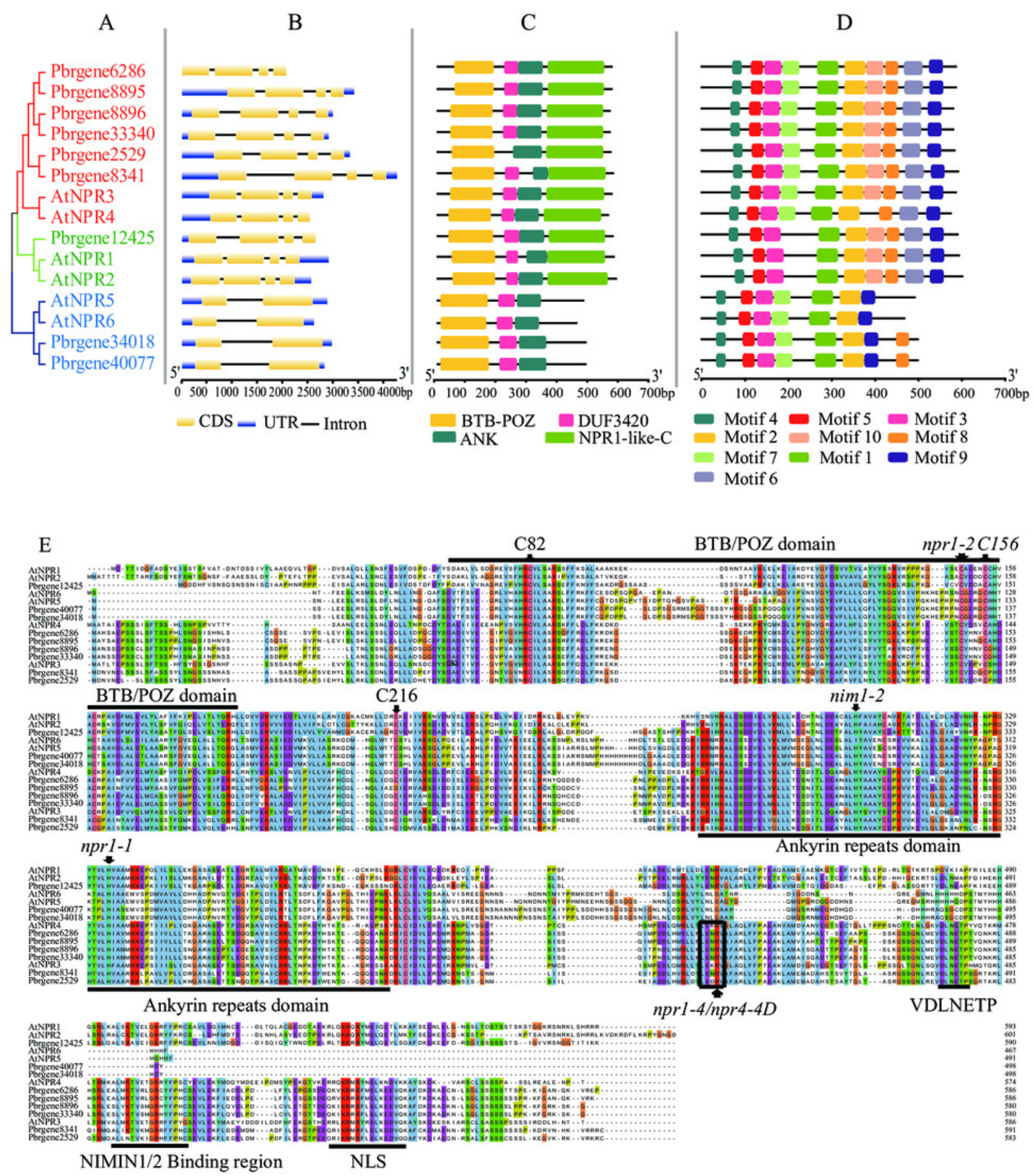
Figure 3

Statistics of cis-acting element numbers in NPR1-like gene family of pear. The different numbers and colors of the grid demonstrated the numbers of different class promoter elements in these genes.

The different numbers and colors of the grid demonstrated the numbers of different class promoter elements in these genes.

\begin{tabular}{|c|c|c|c|c|c|c|c|c|c|c|c|c|c|c|c|c|c|c|c|c|c|c|c|c|c|c|c|c|c|c|c|c|c|c|c|c|}
\hline 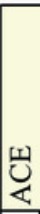 & & & 害 & & & & & & & & & & & نَّ & 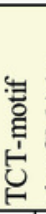 & & & & & & & & & & 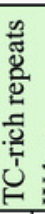 & & 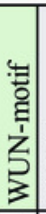 & 宸 & 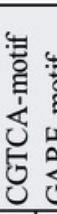 & 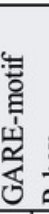 & & 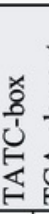 & & 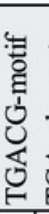 & 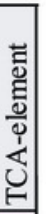 & \\
\hline & & 1 & & & & & 4 & & & & & 1 & & & & & & 1 & 2 & 2 & & 6 & 1 & & & & 1 & \begin{tabular}{|l|l|}
2 & \\
\end{tabular} & \begin{tabular}{l|l}
1 &
\end{tabular} & & & & & 1 & 2 & Pbrgene 12425 \\
\hline & 2 & & 1 & & 1 & & & 3 & & & & 1 & & 1 & 2 & & 1 & 3 & 4 & & & 9 & 3 & 4 & & 1 & & & \begin{tabular}{l|l}
3 & 1 \\
\end{tabular} & 1 & & & & 3 & 2 & Pbrgene 6286 \\
\hline & 1 & & & 1 & & 4 & & & & & & & & 1 & 1 & 1 & 4 & 4 & 1 & & 4 & 3 & 3 & & 1 & 1 & 3 & 4 & & 3 & & & 4 & 3 & & Pbrgene 8895 \\
\hline 1 & & 1 & & & 2 & 1 & 4 & & & & 1 & & & & 1 & & 1 & 3 & 1 & 2 & 1 & 1 & 5 & 3 & 1 & 2 & & 5 & 3 & & & 1 & & 3 & 2 & Pbrgene 8896 \\
\hline & & & 1 & 4 & & 2 & 5 & & & & & & 2 & & & & 2 & 3 & 2 & 3 & 13 & 5 & 2 & & 1 & & 4 & 2 & & 2 & & 1 & 2 & 1 & & Pbrgene2529 \\
\hline & & & & 3 & & 3 & 4 & & & & 1 & & 1 & & & & 4 & 3 & & 3 & \begin{tabular}{l|l}
6 \\
\end{tabular} & 1 & 3 & & 2 & & 2 & 4 & & & & & 4 & & & Pbrgene 8341 \\
\hline & 1 & & & & 4 & & & & & 2 & 1 & & & & 1 & & & 1 & 2 & & 1 & 3 & 4 & 6 & 1 & 1 & 1 & & 1 & & 1 & 1 & & 1 & 4 & Pbrgene 34018 \\
\hline & 1 & & & & 4 & & 1 & & & 2 & 1 & & 1 & & & & & 1 & 2 & & & 5 & 5 & 5 & & 1 & 1 & 1 & 1 & & 1 & & & 1 & 3 & Pbrgene 40077 \\
\hline & & & & & 2 & & 4 & & 1 & 1 & 1 & & & & 2 & & & 3 & 2 & & & 2 & 6 & 2 & & & & \begin{tabular}{l|l}
4 & \\
\end{tabular} & 3 & & $1 \mid$ & \begin{tabular}{l|l}
1 & \\
\end{tabular} & & 3 & 1 & Pbrgene 33340 \\
\hline & & & & & ant & & wt & & $\mathrm{dd}$ & Dev & velor & & & & & & & & & & re & res & $\mathrm{spo1}$ & nsiv & & & & & Horm & mon & R & Res & $\underline{\underline{\mathrm{s}}}$ & sive & & \\
\hline
\end{tabular}




\section{Figure 4}

Expression profile of the PbrNPR1-like genes in ten different tissues

The expression patterns of eight PbrNPR1-like genes in flower, flower bud, young leaf, mature leaf, young stem and mature stem, young fruit, mature fruit, seed tissues were examined by a qPCR assay. FR: flower; FB: flower bud; YL: young leaf; ML: mature leaf; YS: young stem; MS: mature stem; YF: young fruit; MF: mature fruit; SD: seed; BD: bud. The error bars show the standard deviations of the three independent biological replicates. The same letter shows no significantly difference at $P<0.05$ by Duncan's multiple range test.
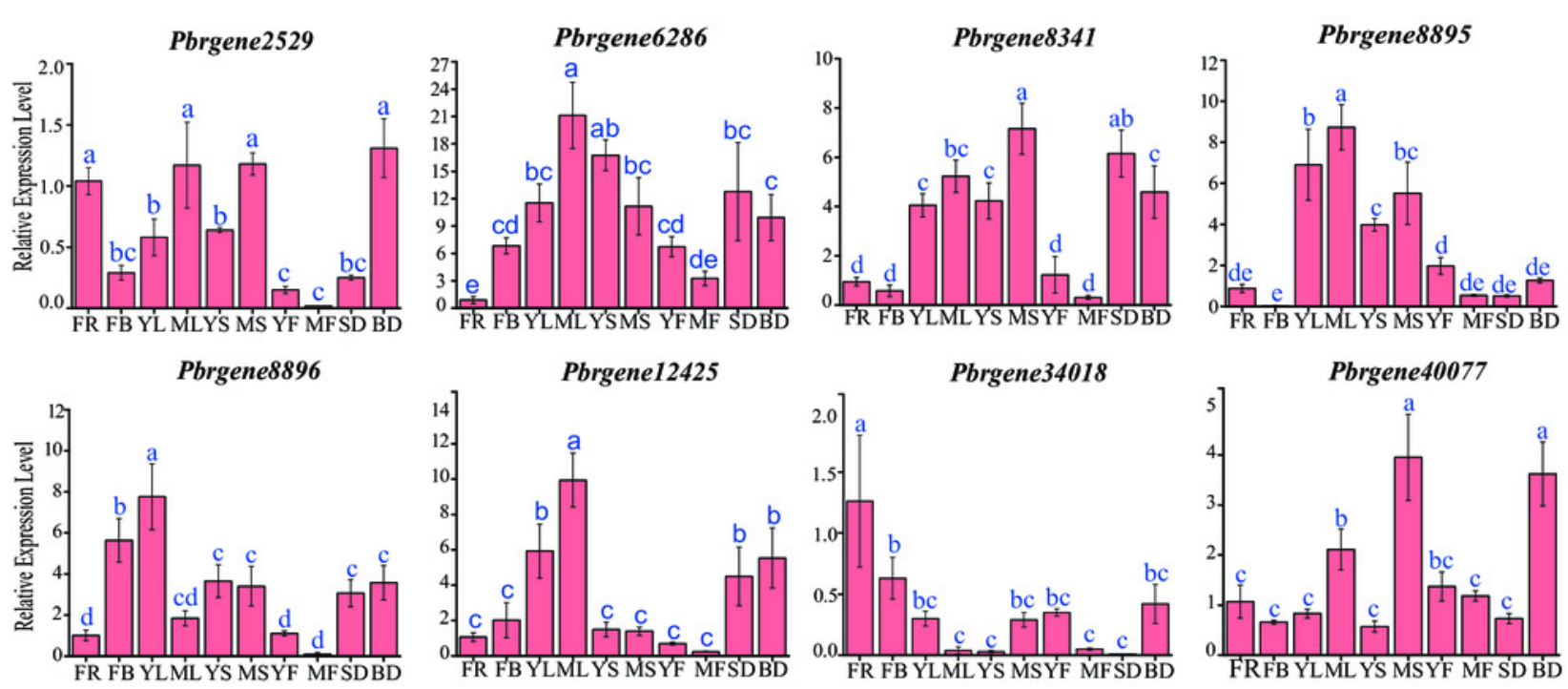
Figure 5

Expression of PbrNPR1-like genes after SA treatment in Yali leaves.

The leaves were harvest at $0 \mathrm{~h}, 1 \mathrm{~h}, 3 \mathrm{~h}, 6 \mathrm{~h}, 12 \mathrm{~h}, 24 \mathrm{~h}, 48 \mathrm{~h}, 72 \mathrm{~h}$ after SA treatment. Data is means $\pm S D$ of $n=3$ biological replicates. The same letter shows no significantly difference at $P<$ 0.05 as determined by Duncan's multiple range test.
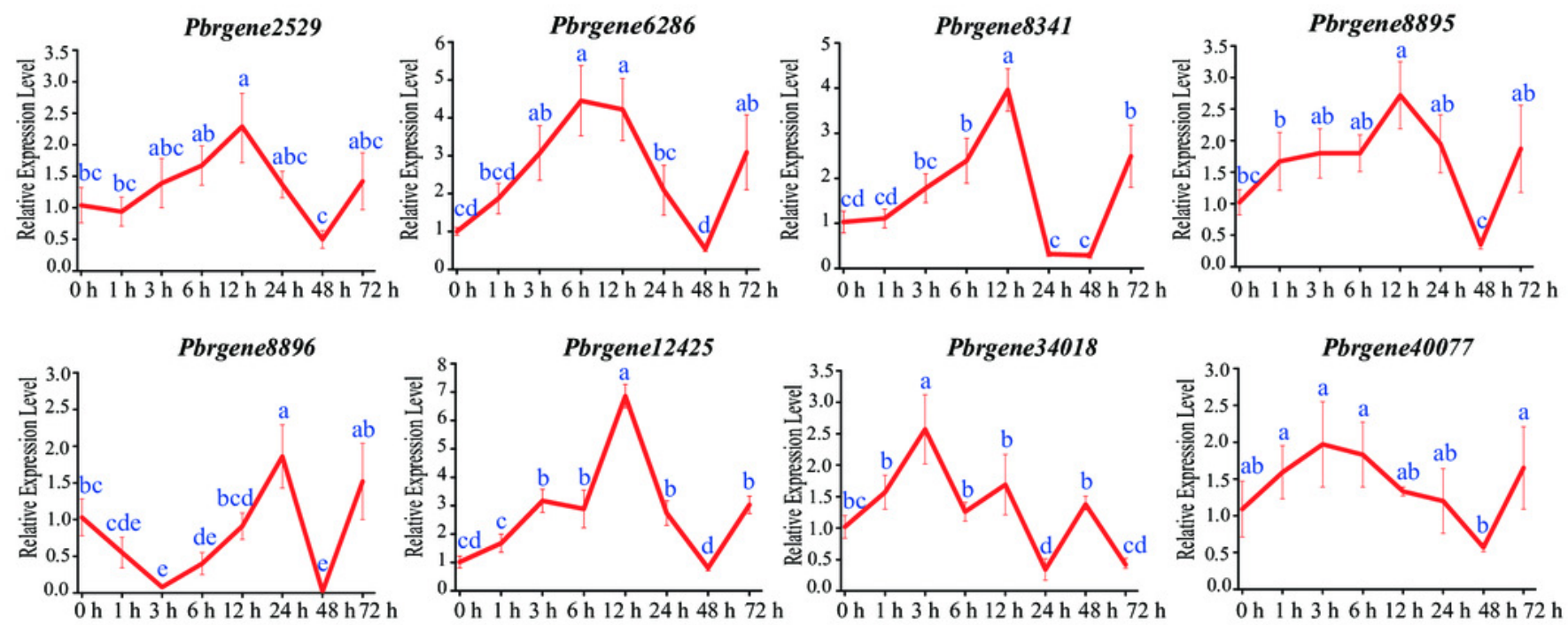
Figure 6

Expression of PbrNPR1-like genes in Yali leaves after inoculation with A.alternata.

The leaves were harvest at $0 \mathrm{~h}, 6 \mathrm{~h}, 12 \mathrm{~h}, 24 \mathrm{~h}, 48 \mathrm{~h}, 72 \mathrm{~h}, 96 \mathrm{~h}, 120 \mathrm{~h}$ after $A$. alternata treatment. Different letters associated with each time point indicate statistically significant differences at the $5 \%$ level. The same letters indicate that the statistics did not differ significantly at $\mathrm{P}<0.05$ according to Duncan's multiple range tests.
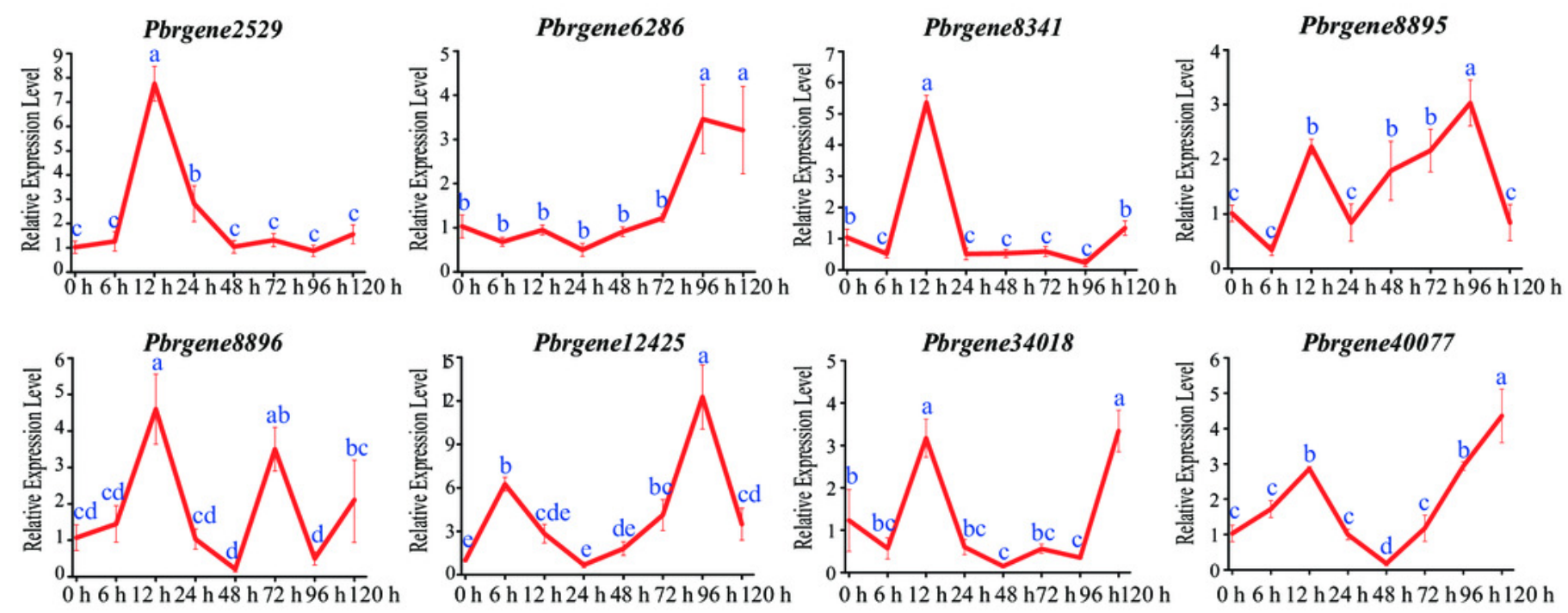
Figure 7

Expression profile of PbrNPR1-like genes in Yali leaves during a day.

The leaves were collected from eight different times of a day on 25 May 2019, 9:00 am, 12:00 am, 15:00 pm, 18:00 pm, 21:00 pm, 24:00, 3:00 am and 6:00 am. Different letters associated with each time point indicate statistically significant differences at the $5 \%$ level. The same the same letters indicate that the statistics did not differ significantly at $\mathrm{P}<0.05$ according to Duncan's multiple range tests.
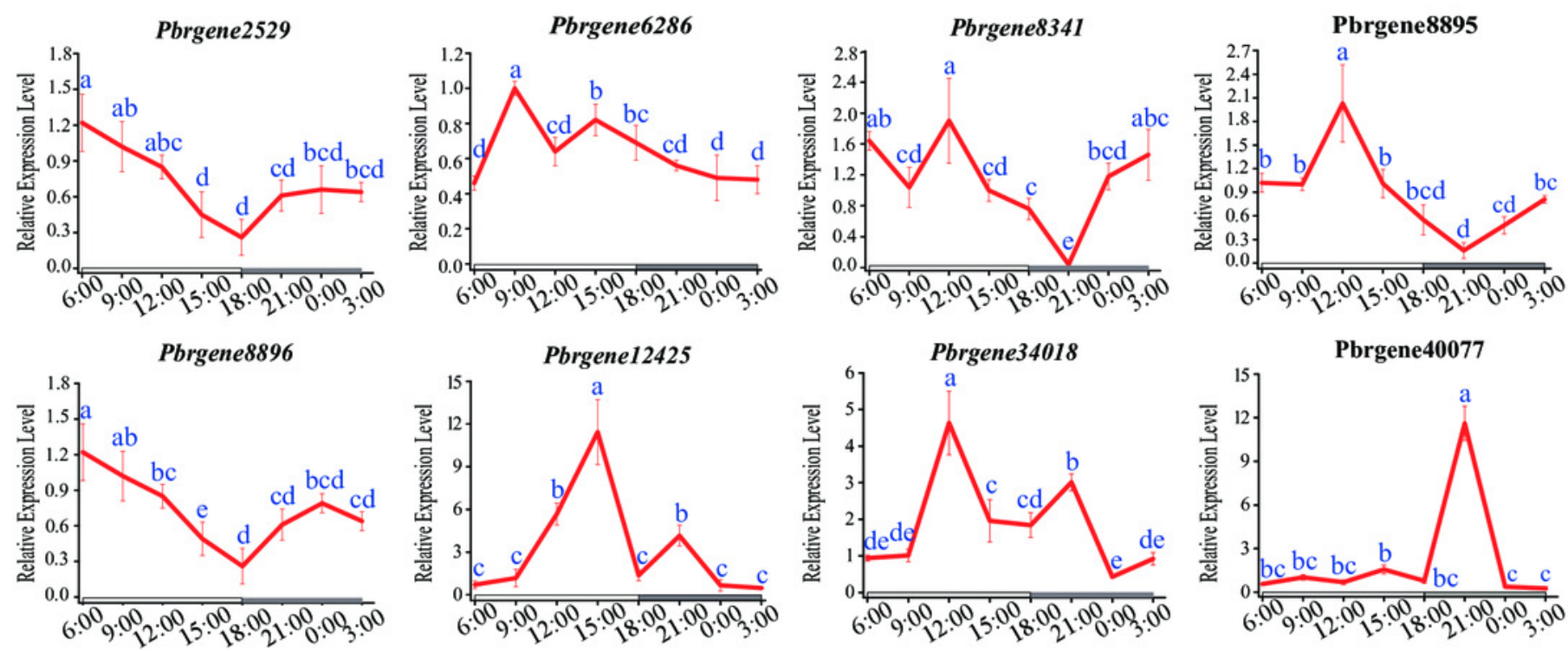